\title{
Le tournage d'un film comme approche à la collecte de matériaux
}

The Shooting of a Film as an Opportunity to Collect Ethnographic Oral Materials СъЁМКИ ФИЛЬМА КАК ПОДХОД К СБОРУ ЭТНОГРАФИЧЕСКОГО И ФОЛЬКЛОРНОГО МАТЕРИАЛА

\section{Denis Kornilov}

Traducteur : Eva Toulouze

\section{OpenEdition \\ Journals}

Édition électronique

URL : https://journals.openedition.org/efo/9786

DOI : $10.4000 /$ efo.9786

ISSN : 2275-1947

\section{Éditeur}

INALCO

Référence électronique

Denis Kornilov, «Le tournage d'un film comme approche à la collecte de matériaux », Études finnoougriennes [En ligne], 49-50 | 2018, mis en ligne le 19 février 2019, consulté le 08 juillet 2021. URL http://journals.openedition.org/efo/9786 ; DOI : https://doi.org/10.4000/efo.9786

Ce document a été généré automatiquement le 8 juillet 2021.

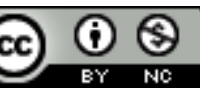

Études finno-ougriennes est mis à disposition selon les termes de la Licence Creative Commons Attribution - Pas d'Utilisation Commerciale 4.0 International. 


\section{Le tournage d'un film comme approche à la collecte de matériaux}

The Shooting of a Film as an Opportunity to Collect Ethnographic Oral Materials

СЪЁМКИ ФИЛЬМА КАК ПОДХОД К СБОРУ ЭТНОГРАФИЧЕСКОГО И ФОЛЬКЛОРНОГО

МАТЕРИАЛА

Denis Kornilov

Traduction : Eva Toulouze

1 La collecte de matériaux de terrain est une dimension indissociable du travail des ethnographes et des folkloristes. Les matériaux collectés par voie d'observation et d'étude directe de la réalité vivante, sont ce qu'on appelle des matériaux de terrain, et le travail de collecte a été appelé travail ethnographique de terrain (Gromov, 1966, p. 4), travail au cours duquel les chercheurs mettent en œuvre diverses méthodes.

2 Dans cet article, je vais essayer de décrire une expérience de création cinématographique fondée sur la production de matériaux vidéo porteurs d'informations ethnographiques. J'ai fait récemment un court-métrage de fiction avec des éléments de cinéma documentaire : les dialogues des acteurs n'étaient pas inscrits dans le scénario, ils étaient improvisés. Je me suis contenté d'indiquer le thème de la conversation.

3 Le sujet du film est simple : une mère (jouée par une habitante du village de KaramasPel'ga dans le raïon de Kijasovo) vit seule au village. Son fils vit et travaille en ville. Cette vieille dame, Luker'ja Sobinova dite Luker'japaj ${ }^{1}$, reçoit la visite d'un voisin, Petyr (A. Vahrušev, professeur à l'université d'Iževsk), qui lui apprend que son fils a l'intention de venir au village. Ravie, elle prépare le plat préféré de son fils, des $k_{y s t y b e j}{ }^{2}$. Mais quand tout est prêt, Petyr lui apprend que son fils ne peut finalement pas venir, il a été appelé au travail de toute urgence. Dans la deuxième partie du film, qui a lieu en été-la première partie avait été filmée en hiver-le fils arrive inopinément avec sa femme. Dans la séquence suivante, ils sont à la maison, à table. Ils ont la visite d'une voisine, Džakyapaj ${ }^{3}$, ils mangent et ils boivent, ils chantent. 
Finalement, la deuxième partie a été exclue de la version définitive (https://youtu.be/ QgG6ug6QPsY).

4 Le rôle principal est joué par Luker'ja Sobinova. Elle vit effectivement seule, mais, à la différence de la Luker'japaj du scénario, elle n'a pas d'enfants. Toutefois elle a joué ce rôle avec un tel naturel que beaucoup de spectateurs ont perçu ce film comme un film documentaire.

En tournant ce film, j'ai relevé bien des faits intéressants (il faut noter que mon objectif était de faire un film, par un article!). Tout d'abord, l'improvisation des dialogues a réellement été un succès, les acteurs on fait appel à des proverbes, des dictons, qui se sont révélés une découverte pour moi. Deuxièmement, dans bien des scènes où Luker'japaj est seule, elle a oublié que je tournais et s'est mise à raconter sa vie et à exprimer son opinion sur telle ou telle situation. J'ai été particulièrement intéressé par ses dialogues avec les objets et avec les animaux.

Je voudrais commencer par relater quelques moments intéressants du tournage. Prenons d'abord les scènes de solitude.

Pendant la scène où elle allume le poêle, elle a présenté une belle comparaison. Elle a comparé l'absence de poêle dans la maison avec une maison ou une famille sans mère :

\begin{abstract}
Э-Э-Э, АЗЬЛО ТАЗЬЫ ЧАГ КЕСЬЫСА ГУР ВЫЛЭ ПОНОНО ВАЛ, КЕРТТЫЛЫСА, ТОЖО. ТУАЛА ПИНАЛЪЁСЛЭН ЧАГ КЕСЕМЗЫ НО УГ ПОТ НИ, ГАЗЕТЭН СУТО. КУДӤЗЛЭН ГУРЕЗ НО ӦВӦЛ НИ. ГАЗЛЫ ОСКЫСА. ГАЗЛЫ ОСКОНЭД, ТОЖЕ, ГУРТЭК КЕЛЬТЫСА, КОРКАН АНАЙТЭМ КАДЬ КЫЛЕ СО, ГУР КЕ ӦВӦЛ. ГУРЕЗ КЕСЯН УГ ЯРА.

Eh-eh-eh, cela arrivait qu'on prépare les brindilles à l'avance et qu'on les mette dans le poêle. Aujourd'hui, les jeunes ne veulent même plus préparer des allumefeu, ils font $d u$ feu avec du papier. Et certains n'ont même plus de poêle. Ils comptent sur le gaz. Sans poêle, une maison est comme sans mère. Il ne faut pas démonter le poêle.
\end{abstract}

Ce média ne peut être affiché ici. Veuillez vous reporter à l'édition en ligne http:// journals.openedition.org/efo/9786

Dans cette même scène, nous avons une formule incantatoire :

ГОРИ, ГОРИ ЯСНО, ЧТОБЫ НЕ ПОГАСЛО» НО ШУСА ВЫРИСЬКО АС СЯМЕНЫМ. ДАВАЙ, ЭН КЫСЫ. МУРЪЁЭТЙ ӴЫНЫЗ МЕД ПОТОЗ, ШУНЫТЭЗ КОРКА МЕД ПОТОЗ, МОНЭ МЕДАЗ КЫНТЫ.

Brûle, brûle bien clairement, pour qu'il ne s'éteigne pas $»^{4}$. C'est aussi ce que je me dis... Allez, ne t'éteins pas. Que la fumée parte par la cheminée, qu'il fasse chaud dans la maison, pour que je n'aie pas froid.

Ce média ne peut être affiché ici. Veuillez vous reporter à l'édition en ligne http:// journals.openedition.org/efo/9786

Luker'japaj propose une autre comparaison intéressante :

ОЙ, ЖАДИСЬКО УГОСЬ ТАНИ. ПУКСЬЫСА ПОГЫЛЬТӤСЬКЫН УГ ЛУ. ТЫБЫРЫ ЖАДЕ. ӜАКЫ АПАЙ СО ШЫР КАДЬ ШАПЛЫ АЛИ. ПЫД УЛАЗ ШЫР КЕ НО СЮРИЗ, КУЛЫСА УЗ ВУ, ПЕГӞОЗ ШЫР. ШАПЛЫ ВАМЫШЪЯ АЛИ СО.

Oh, c'est que je fatigue. Je ne peux plus faire la pâte assise. Le dos fatigue. Džaky apaj. - Elle est encore comme une rapide petite souris. Si une souris se retrouve sous ses pieds, elle n'a pas le temps de mourir, la souris s'enfuit. Elle marche encore très vite. 
Ce média ne peut être affiché ici. Veuillez vous reporter à l'édition en ligne http:// journals.openedition.org/efo/9786

Les dialogues présentés ci-dessous contiennent également beaucoup de formules intéressantes du point de vue linguistique et folkloristique :

— ПЫРЫН ЯРА-А МАР-А ӜАКЫ АПАЙ? 'МОЖНО ЛИ ЗАЙТИ, ӜАКЫ АПАЙ?'

- On peut entrer, Džaky apaj ?

— ЯРА, ЯРА, МАЛЫ УЗ ЯРА ПЫРЫНЫ?! Ӧс ПЫРЫНЫ ТУПАТЭМЫН УК.

- Oui, bien sûr, pourquoi ne pourrais-tu pas rentrer ? C'est à cela que sert la porte.

- ОЗЬЫ. КАПКА НО... 'ЭТО ТАК. ВОРОТА ТОЖЕ...'

- C'est bien cela. Le portail, c'est pareil.

- ПУКСьЫ АЛИ, ПУКСЬЫ, ПУКСЬЫ. ПУКСЬЫ, ВЕТЛЭМЕД КЕМА ВАЛ ИНИ.

- Vas-y, assieds-toi ! Assieds-toi, cela fait longtemps que tu n'es pas venue.

- МУГЕН ГИНЭ ПОТАСЬКО, КАЛТЫРТЭМ УГ ПОТЫ. КАЛТЫРТЫН КЕ ПОТӤСЬКО - КЫЛЫ КУЗЬ, МИНДЭРЕ НЕБЫТ ЛУЭ.

- Je ne sors que s'il le faut, autrement, je ne veux pas. Si je ne sors que comme ça, ma langue s'allongera, mon oreiller deviendra mou.

Ce média ne peut être affiché ici. Veuillez vous reporter à l'édition en ligne http:// journals.openedition.org/efo/9786

Le discours de Luker'japaj est farci de phraséologie. Dans la conversation sur la télévision qui ne fonctionne pas, elle le reproche aux réparateurs et prétend qu'ils aiment boire et ne comprennent rien à rien :

- ЧАРКА ЯРАТЙСЬ МУРТ СО, СЫӴЕ ТЕЛЕВИЗОР КОТЫРЫН УГГЕС ПӦРМЫТЫ КАДЬ.

- Ceux qui aiment l'alcool, semble-t-il, ne sont pas capables de réparer les équipements.

Ce média ne peut être affiché ici. Veuillez vous reporter à l'édition en ligne http:// journals.openedition.org/efo/9786

- СЕКЫТ ӦВӦЛ СО НО. ЧАРКА ДУРЕ УСИСЬКОД КЕ, СО ПУНЬЫДЭ ӜУУТЫНЫ НО, АСТЭ КӦТ ТЫРЫНЫ НО УГ ЛУЫ НИ СОИН.

- Ce n'est pas difficile. Mais si on tombe à côté d'un petit verre, et on lève la cuillère, on n'arrive plus à se nourrir.

Ce média ne peut être affiché ici. Veuillez vous reporter à l'édition en ligne http:// journals.openedition.org/efo/9786

Le caractère expressif de son discours se voit bien dans ses réflexions sur la vie, dans ses souvenirs :

- МОН ПИНАЛЪЁСЛЫ УЛОН АЛАМА МЕДАЗ КЫЛЬ ВАЛ ШУСА УЛӤСЬКО. МИ АДӞИМ ИНИ НО, БУДӤСЬ ПИНАЛЪЁС ТАНИ УЖТЭК КЫЛЕМЫН, КОЛХОЗ БЫРИЗ, ОЗЬЫ-ТАЗЬЫ УРАМ ВОЛЯТЫСА ГИНЭ НА, ТИНИ ОЗЬЫ-ТАЗЬЫ ЧАРКА ДУРЕ УСЬКЫТӤСЬКЕМЫН. ВОТ, ПИНАЛЪЁС АСЬСЭ ПОННА УЛЫСА, УЖТЭК КЫЛЬЫСА, МАР УЖАНЫ ӞУДЫСА, ПИНАЛЪЁС ВОЙНА МЕДАЗ ПОТТЭ АСЬСЭ ПОННА, ШУЫСА, СЮЛЭМ ВИСЕ ТӤнИ СОЛЫ. ‘

- Je voudrais que les jeunes ne se retrouvent pas avec une mauvaise vie. Nous avons beaucoup vu. Les jeunes qui grandissent se retrouvent sans travail, le kolkhoze s'est désagrégé, eux ne font qu'égaliser les rues [traîner dans l'oisiveté], s'affaler près du petit verre [boire]. Oui, que les jeunes, sans travail, ne sachant pas 
quoi faire, qu'ils ne déclenchent pas eux-mêmes une guerre. Voilà ce qui me fait mal au cœur.

Ce média ne peut être affiché ici. Veuillez vous reporter à l'édition en ligne http:// journals.openedition.org/efo/9786

Tout prétexte au dialogue donne l'occasion d'évoquer le passé et imperceptiblement, d'inscrire dans la conversation des phraséologismes et des expressions figées :

— ПЕНСИЕЗ МЕДАЗ БЫДТЭ ШУСА КУРДАСЬКО МОН.

- J'ai peur qu'ils ne cessent de verser les retraites.

- УЗ, УЗ, МА КЫЗЬЫ ПЕНСИЕЗ АЛИ... УЗ БЫДТЭ.

- Non non, comment peuvent-ils arrêter. Non, ils ne le feront pas.

- МА, КИН ТОДЭ. УЛОН АЛАМА ЛУИЗ КЕ, ВСЁ. БАРАНГИ МЕД СИЁЗЫ ШУОЗЫ. АЗЬЛО КРАХМАЛ

СИИМ, БАРАНГЫ СИИМ. АТАЕ ШУОЗ ВАЛ: «ТА БАРАНГИ ОШИСЬКЫСАЛЫЗ ИНИ: НЯНЬ ИНТЫЕ НО, ӞЕГ НЯНЬ ИНТЫЕ НО БАРАНГЫ СИИСЬКОМЫ, АС ИНТЫЯЗ НО СИИСЬКОМЫ, ВС̈̈ БАРАНГЫ»,- ШУОЗ ВАЛ.

- Qui sait. Si la vie devient pire, c'est fini ! Ils disent : «ils n'ont qu'à manger des pommes de terre ». Avant on mangeait des féculents, des pommes de terre. Parfois, mon père disait : "Cette pomme de terre pourrait aller se faire pendre ! À la place du pain, [on mange des pommes de terre], à la place du pain de seigle, on mange des pommes de terre et à la place des pommes de terre, on mange des pommes de terre, rien que des pommes de terre ", voilà ce qu'il disait.

Ce média ne peut être affiché ici. Veuillez vous reporter à l'édition en ligne http:// journals.openedition.org/efo/9786

- ПУРТЭД НО МАР-КЕ УМОЙ-УМОЙ УГ БАСЬТЫ НИ. ШЕРСА КЕЛЬТО АЙ. ‘

- Le couteau ne coupe plus bien. Il va falloir l'affûter.

- АЧИМ НО НЫЖОМЕМЫН УГОСЬ ИНИ. УЖЛЫ ЧАЛЯК ӦВӦЛ НИ. ПЫД УГ МЫН НИ, КАНЖАНО ГИНЭ НА. ПУРТЛЫ НО ШЕР КУЛЭ УК. ЗАРНИ КИОС КУЛЭ КОТЬМАЛЫ.

- Moi-même, je suis émoussée. Je ne peux plus travailler rapidement. Mes jambes n'avancent pas, je ne fais que trébucher. Il fallait aussi que le couteau s'émousse. Pour tout, il faut des mains en or.

Ce média ne peut être affiché ici. Veuillez vous reporter à l'édition en ligne http:// journals.openedition.org/efo/9786

Nous avons rencontré une situation intéressante quand Luker'japaj est descendue à la cave. Dans le premier dialogue, à la question de savoir ce qu'elle y cherchait, elle répond que le chat y avait déféqué, et qu'elle nettoyait. Tout le monde a compris cette phrase au sens propre. Mais au cours du tournage, dans une autre scène, elle est revenue sur cette expression (voir le deuxième dialogue) :

- МАРО-О УТЧАСЬКОД НОШ ОТЫН? МАР-О ПОТТЫНЫ?

- Qu'est-ce que tu cherches là ? Qu'est-ce que tu en rapportes ?

- ПИСЭЙ СИЧАСЬКЕМ НО, СОЕ ОКТЫНЫ ПЫРИ АЛИ.

- Le chat a déféqué, je suis allée nettoyer.

$-M A P ?$

- Quoi?

- ЗЫН МЕДАЗ ПОТЫ ШУСА, ПИСЭЙ СИЧЕЗ ОКТЫНЫ ПЫРИ АЙ. '

- Je suis allée nettoyer le caca du chat pour éviter des mauvaises odeurs.

- A-A-A. Il vient par-là, hein, l'été aussi ? Chasse-le! 

uns. Ainsi, la plupart d'entre eux ont été des improvisations sur un thème donné. Mais certains dialogues ont requis un travail préalable. Il a fallu sur place réfléchir à ce qu'ils allaient dire. Dans ce processus, Luker'japaj a été de bon gré active et elle a proposé des phrases d'une grande sagesse. Ci-dessous, quelques exemples :

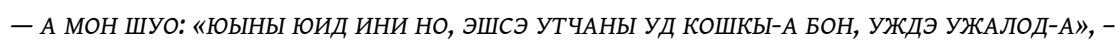
ШУОМ-А?

- Alors moi je dis : « pour ce qui est de boire, tu as déjà bu, mais tu ne vas pas aller chercher une copine à ton petit verre, est-ce que tu vas terminer le boulot... » Je peux dire comme ça?

- АГА, АГА, ШУЭ, ШУЭ ОЗЬЫ.

- Oui, oui, dites cette phrase !

- озьы шуо.

- Eh bien c'est ce que je vais dire.

- НУ ЛАДНО, ТАЗАЛЫК ПОННА, ЯВА, ЛУКЕРЬЯПАЙ.

- C'est bon, c'est bon, à votre santé, Luker'japaj.

- Oui, c'est bon. Pour ton aide!

- ОХ, ЧЕСКЫТ КОШКИЗ.

- Ah, c'était bon!

- ЧЕСКЫТ ШУЫНЫ, ПЕ, УГ ЯРА.

- Il paraît qu'il ne faut pas dire « bon » [pour de l'alcool].

- ОзьЫ шуоно-А, ПЕ, ӧвӧл?

- Ah bon, il ne faut pas, hein?

- ДА. А ТО, ЧЕСКЫТ КЕ ШУИД, МУКЕТСЭ УТЧАНЫ КОШКОНО ЛУЭ.

- Oui, si on dit qu'il est bon, il faut aller chercher une autre bouteille.

- ДА, ОЛОМАР НО ВЕРАЛОД ТОН.‘

- Ah, que ne racontes-tu pas!

Ce média ne peut être affiché ici. Veuillez vous reporter à l'édition en ligne http:// journals.openedition.org/efo/9786

- ОЙДОЛЭ, СОКУ ОЗЬЫ ШУОМЫ-А. ТӤНИ СО СЯРЫСЬ ВЕРАСЬКОМЫ. «ВОЙНА ГИНЭ МЕДАЗ ПОТЫ НИ ВАЛ СО», - ШУЭЛЭ ТӤ. «ПИНАЛЪЁС МЕДАЗ АДӞЕ ВАЛ НИ, МАЕ МИ АДӞИМЫ», ШУОДЫ, ПЕТЫР ШОРЫ УЧКЫСА.

- Allez, on va dire comme ça. Voilà, on en parle. Vous dites : «Pourvu qu'il n'y ait plus de guerres ». Et vous dites : «Que les jeunes ne voient pas ce que nous avons dû vivre », en regardant Petyr. Et vous, Mihail Petrovič, vous dites, qu'il n'y aura plus 
de grande guerre. Vous dites que les grandes guerres ne sont pas profitables, il n'y aura que des guerres locales.

- СО ОЗЬЫ ШУИЗ КЕ, МОН, «ВОЙНАЛЭН МАР КЕ ВЫГОДНО-А МАР-А, КЫТЧЫ КЕ ЗАРНИ КИСЬТЫСА КЕЛЬТО-А МАР-А БОМБАЫСЬТЫЗЫ», ШУО. '

- Et quand lui il dit ceci, moi, je lui dis : « pourquoi, une guerre, cela rapporte, avec les bombes, ils vous jettent de l'or ", que je lui dis.

- умой, умой, о-о.

- Oui, oui, c'est bien.

- Я... ТАРЕ ВЕРАСЬКОМ-А НИ? ‘

- Bien... Maintenant il faut parler?

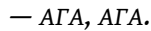

- Oui, oui.

- ПЕТЫР, МОН СОЛЭСЬ ТУЖ КУРДАСЬКО, ВОЙНА ГИНЭ МЕДАЗ ПОТЫ НИ. ВОЙНАЛЭСЬ КУРДАСЬКО. МИ СИЫТЭК НО, ДЙСЬКУТТЭК НО БУДИЙМ БЕРЕ, О-О, ТУЖ СОЛЭСЬ КУРДАСЬКО НО, ВОЙНА ПОТОНО ЛУИЗ КЕ, ПЕНСИЕЗ НО БЫДТОЗЫ. МИ СИЫТЭК УЛӤМ, СОБЕРЕ ВОКСЁ сиытэК кУломы.

- Petyr, pourvu qu'il n'y ait pas de guerre. C'est de ça que j'ai peur. J'ai peur de la guerre. Nous, on a connu la faim et on n'avait rien à se mettre, j'ai très peur, qu'il y ait une guerre et qu'ils ne versent plus les retraites. Nous on a grandi avec la faim. Et puis on mourra de faim.

- ЛУКЕРЬЯПАЙ, БАДӞЫМ ВОЙНА АЛИ УЗ ЛУЫ НИ. МИХАИЛ ПЕТРОВИЧ, ДЫШЕТӤСЬ ШКОЛАЫСЬТЫМЫ, ШУЭ, АЛИ, ПЕ, СО НЕ ВЫГОДНО, АЛИ, ПЕ, ВЫГОДНО ЛОКАЛЬНОЙ... ЛОКАЛЬНОЙ ВОЙНА ГИНЭ НУЫНЫ, ПЕ, ВЫГОДНО. А БАДӞЫМ ВОЙНА, ПЕ, АЛИ ВЫГОДНО ӦВӦЛ НИ, ПЕ.

- Luker'japaj, il n'y aura plus de grande guerre maintenant. Mihail Petrovič, l'instituteur, dit que cela ne rapporte rien, maintenant, dit-il, seules les guerres locales rapportent. Mais les guerres globales, dit-il, cela ne rapport plus rien.

- А ВОЙНАЛЭН МАР ВЫГОДНОЕЗ БОН, ПЕТЫР? МАР СЁТЭ ВЫГОДНОЙ? БОМБАЫСЬТЫЗЫ ЗАРНИ КИСЬТЫСА КЕЛЬТО-А МАР-А ВОЙНА ЫБЫЛӤСЬКЕМ БЕРВЫЛАЗЫ?

- Mais qu'est-ce que ça rapporte, une guerre, Petyr? Quel profit? Il sort de l'or des bombes sur les champs ou quoi?

- О-О, ТАНИ ТУЖ УМОЙ ВЕРАД, АГА. ВАНЬ СЫӤЕ АДЯМИОС, УЖБЕРГАТӤСЬЁС, ОЛИГАРХЪЁС ВСЯКОЙ, КУДъЁСЫЗЛЫ ВОЙНА ТУЖ ВЫГОДНО, ЗАРНИ ПОТТО ОТЫСЬ. АДЯМИ КУЛЭ, А СООС ЗАРНИ ПОТТО. СЫӴЕОСЫЗ НО ВАНЬ... КЫЗЬЫ-О ШУО: КОМУ ВОЙНА, КОМУ МАТЬ РОДНА. ОЗЬЫ ВЕРАЛО ӞУЧЪЁС, О-О. ВОТ СОИЗ ОТЫН ВАНЬ, ЗЭМ ВЕРАМЫН СО. '

- Oui, vous avez très bien dit, ahaa. Il y a des gens, des hommes d'affaires, toutes sortes d'oligarques, à qui la guerre rapporte beaucoup, ils en forgent de l'or. Les gens meurent, mais eux, ils en font de l'or. Comme on dit, à qui la guerre, à qui la mère. C'est ce que disent les Russes. C'est bien dit, oui.

- À qui les os et à qui l'or, oui ? À qui les os et à qui l'or?

- ВОТ САМОЙ ОЗЬЫ, О-О, ВОТ ШОНЕР ВЕРАСЬКОД. ВОТ ОЗЬЫ...

- Oui, c'est comme ça, tu dis vrai, voilà'.

Ce média ne peut être affiché ici. Veuillez vous reporter à l'édition en ligne http:// journals.openedition.org/efo/9786

La situation de tournage a permis aux participants au projet de figurer sous divers avatars: le «jeu de rôles» leur a fourni des cadres, et leur permettant de rester euxmêmes. Et c'est l'expérience de la vie déjà vécue, les connaissances personnelles qui ont été le fondement sur lequel ont été construits les dialogues naturels entre les protagonistes à l'aide de formules qui, en cas de notation standard, auraient pu ne pas venir à l'idée. Mon expérience du filmage a confirmé une fois de plus l'idée des ethnographes, d'après laquelle il est important de ne pas «verrouiller l'initiative du 
narrateur et ne pas se priver ainsi des nombreuses intéressantes informations qu'il pourrait transmettre dans le cadre d'une narration libre et active " (Gromov, 1966, p. 26-27).

Il convient de se laisser guider par la vérité relevée par différentes générations de chercheurs spécialistes de la culture et des pratiques des différents peuples sous toutes leurs formes :

Observez les phénomènes ethnographiques avec la même minutie, précision, exhaustivité et objectivité que le naturaliste qui observe les phénomènes et les éléments de la nature. Constatez tout ce qui s'offre à l'observation, sans faire intervenir de subjectivité dans le partage des phénomènes en significatifs et non significatif, puisque souvent ce qui semble être non significatif ou peu significatif pour avoir une très grande signification pour une approche comparative. (Sternberg, 1914, p. 212)

Dans les conditions du terrain, les ethnographes ont à faire à une réalité vivante, en mouvement permanent. Il est impossible de prévoir à l'avance toute la diversité des éléments qui, dans une mission, mériteront d'être étudiés. On ne peut pas faire de recommandations, proposer des méthodes qui conviendront à tous les cas qui risquent de se présenter. Suivant les conditions concrètes, il va falloir choisir les moyens d'étude les plus efficaces, voir inventer de nouvelles méthodes. Seule une approche créative permet de réussir à remplir les tâches de l'ethnographe pendant le terrain (Gromov, 1966, p. 39).

\section{BIBLIOGRAPHIE}

GROMOV G. ГРОМОВ Г., 1966, МЕТОДИКА ЭТНОГРАФИЧЕСКИХ ЭКСПЕДИЦИЙ, [La méthode des travaux de terrain ethnographiques], изД-Во московского унивЕРСиТЕТА [Presses Universitaires de Moscou], Москва [Moscou], 108 p.

STERNBERG L. Ya. ШТЕРНБЕРГ Л. Я., 1914, КРАТКАЯ ПРОГРАММА ПО ЭТНОГРАФИИ (ПРИМЕНИТЕЛЬНО К БЫТУ СЕВЕРНЫХ ИНОРОДЦЕВ). СБОРНИК ИНСТРУКЦИИ И ПРОГРАММ ДЛЯ УЧАСТНИКОВ ЭКСКУРСИЙ В СИБИРь, [Bref programme d'ethnographie (appliqué à la vie des autochtones du Nord). Recueil d'instructions et de programmes pour les participants aux expéditions en Sibérie], САНКТ ПЕТЕРБУРГ [Saint-Pétersbourg].

\section{NOTES}

1. En oudmourte, АПАЙ, араj, litt. sœur aînée, est le titre respectueux qu'on donne aux femmes âgées.

2. En oudmourte : КЫстыБЕй, galettes recouvertes de purée de pommes de terre ou de bouillie de céréales.

3. Surnom d'Olga Solovjova.

4. En russe. Le reste de la phrase est en oudmourte. 


\section{RÉSUMÉS}

Cet article montre comment la création d'un court-métrage oudmourte de fiction a permis d'obtenir des informations ethnographiques d'oralité spontanée. En 2015, j'ai tourné un film dans le village de Karamas-Pel'ga du raïon de Kijasovo en République d'Oudmourtie. J'avais écrit un scénario à l'avance, mais sans écrire les dialogues dans le détail. Ainsi, lors du tournage, je me suis contenté de dire aux acteurs de quoi ils étaient censés parler. Les dialogues, en fin de compte, ont été improvisés et ils se sont avérés riches en tournures intéressantes. L'idée d'écrire un article à ce sujet a émergé lors de l'analyse et du travail sur les rushes vidéo, qui m'ont permis de découvrir la richesse de ces informations d'oralité ethnographique. Cet article me donne l'occasion d'en livrer quelques-unes.

This article shows how the shooting of a short Udmurt fiction film gave the opportunity to obtain some spontaneous ethnographic oral information. In 2015 I shot a film in the village of KaramasPel'ga, in the district of Kiyasovo in the Udmurt Republic. I had written beforehand a scenario, but without having all the dialogues written in extent. So, while shooting, I just informed the actors of what they were supposed to talk about. Eventually the dialogues were improvised and elicited a huge amount of rich and interesting expressions. This idea of writing an article about these questions emerged while I analysed and worked on the video material. This article give me the opportunity of presenting some of my discoveries.

СТАТЬЯ ПОСВЯЩЕНА ОПИСАНИЮ ОПЫТА СОЗДАНИЯ УДМУРТСКОГО КОРОТКОМЕТРАЖНОГО ИГРОВОГО ФИЛЬМА КАК ОСНОВЫ ПОЛУЧЕНИЯ СПОНТАННОЙ ФОЛЬКЛОРНО-ЭТНОГРАФИЧЕСКОЙ ИНФОРМАЦИИ. В 2015 ГОДУ МНОЙ БЫЛ СНЯТ ФИЛЬМ В ДЕРЕВНЕ КАРАМАС-ПЕЛЬГА КИЯСОВСВКОГО Р-НА УДМУРТСКОЙ РЕСПУБЛИКИ. Я ЗАРАНЕЕ НАПИСАЛ СЦЕНАРИЙ, НО ДИАЛОГИ ТОЧЬ В ТОЧЬ НЕ ПРОПИСЫВАЛ. В ИТОГЕ, ВО ВРЕМЯ СЪЁМОК АКТЁРАМ Я ГОВОРИЛ ТОЛЬКО О ЧЕМ НУЖНО ГОВОРИТЬ. ДИАЛОГИ ПОЛУЧИЛИСЬ ИМПРОВИЗИРОВАННЫМИ И БОГАТЫМИ ОБОРОТАМИ РЕЧИ. ИДЕЯ НАПИСАНИЯ СТАТЬИ ПРИШЛА ВО ВРЕМЯ АНАЛИЗА И ОБРАБОТКИ ВИДЕОМАТЕРИАЛА, ВО ВРЕМЯ КОТОРОГО Я НАТКНУЛСЯ НА НЕМАЛОЕ КОЛИЧЕСТВО ФОЛЬКЛОРНО-ЭТНОГРАФИЧЕСКОЙ ИНФОРМАЦИИ. ПРО НЕКОТОРЫЕ ИЗ НИХ, ПОПЫТАЮСЬ РАССКАЗАТЬ В ЭТОЙ СТАТЬЕ.

\section{INDEX}

Mots-clés : Anthropologie visuelle, Anthropologie visuelle, cinéma de fiction, cinéma de fiction, film, film, improvisation, improvisation, informations ethnographiques, informations ethnographiques, XXIe siècle début, XXIe siècle début, Oudmourtes, Oudmourtes, KaramasPel'ga, Karamas-Pel'ga, Kijasovo, Kijasovo

Keywords : Visual anthropology, ethnographic information, fiction film, film, improvisation, 21th century beginning, Udmurt, Karamas-Pelga, Kiyasovo

motsclesru ВИЗУАЛЬНАЯ АНТРОПОЛОГИЯ, УДМУРТСКИЙ ФИЛЬМ, ИГРОВОЕ КИНО, ИМПРОВИЗАЦИЯ, ФОЛЬКЛОРНО-ЭТНОГРАФИЧЕСКАЯ ИНФОРМАЦИЯ, УДМУРТ КИНО, ШУДОН КИНО, ФОЛЬКЛОРЕН НО ЭТНОГРАФИЕН ГЕРӞАСЬКЕМ ТОДОН-ВАЛАН, ХХІ ВЕК НАЧАЛО, УДМУРТЫ, КАРАМАСЬ-ПЕЛЬГА, КИЯСОВО 